\title{
Diversité floristique et caractérisation structurale de la réserve forestière de Ouoghi en zone soudano- guinéenne (Centre-Bénin)
}

\author{
Sfich T. B. Ahouandjinou, \\ Hounnankpon Yédomonhan, \\ Monique G. Tossou, \\ Aristide C. Adomou, \\ Akpovi Akoègninou
}

Laboratoire de Botanique et Ecologie Végétale, Faculté des Sciences et Techniques, Université d'Abomey-Calavi, 01 B.P 4521 Cotonou, République du Bénin

doi: 10.19044/esj.2017.v13n12p400 URL:http://dx.doi.org/10.19044/esj.2017.v13n12p400

\begin{abstract}
Objective: The objective of this study is to identify the plant species and to characterize the vegetation of the Ouoghi forest reserve in the commune of Savè (Center-Benin).

Method and results: Floristic and forest inventories were carried out through 144 relevés in according to the Braun-Blanquet method. A total of 246 plant species were identified. They belong to 178 genera and 63 families. Multivariate analyzes with the Community Analysis Package (CAP) software made it possible to individualize 3 vegetation formations comprising 8 plant groups. This is the shrub savannah with Prosopis africana and Pennisetum polystachion, the tree savannah with Hymenocardia acida and Pennisetum polystachion, the tree savannah to Vitellaria paradoxa and Pennisetum polystachion, the tree savannah to Pterocarpus erinaceus and Hyptis suaveolens, the woodland savannah with Daniellia Oliveri and Chromolaena odorata, the woodland savannah at Isoberlinia doka and Pennisetum polystachion, the woodland savannah at Daniellia oliveri and Andropogon tectorum and the tree flood savannah to Daniellia oliveri and Leersia hexandra. The floristic and structural parameters change from one group to another. Thus, the specific richness varies from 49 to 153 species and the diversity of families is between 22 and 49 with a predominance of Leguminosae. The density varies from 95 to 275 stems / ha, basal area from 2.35 to $8.44 \mathrm{~m}^{2}$ / ha.
\end{abstract}


Conclusion and application of results: The study evaluated the floristic diversity of the Ouoghi reserve. In addition, it indicated the need for its integral protection for its dynamics towards dry dense forest vegetation. Sustainable management of this site needs implication of local population.

Keywords: Phytodiversity, plant communities, vegetation, Sudano-Guinean zone

\section{Résumé}

Objectif : L'étude a pour objectif de recenser les espèces végétales et de caractériser la végétation de la réserve forestière de Ouoghi dans la Commune de Savè (Centre-Bénin).

Méthode et résultats : Les inventaires floristiques et forestiers ont été réalisés à travers 144 relevés effectués suivant la méthode de Braun-Blanquet. Au total, 246 espèces végétales ont été recensées. Elles appartiennent à 178 genres et 63 familles. Les analyses multivariées avec le logiciel «Community Analysis Package» ou CAP ont permis d'individualiser 3 formations végétales comprenant 8 groupements végétaux. Il s'agit de la savane arbustive à Prosopis africana et Pennisetum polystachion, la savane arborée à Hymenocardia acida et Pennisetum polystachion, la savane arborée à Vitellaria paradoxa et Pennisetum polystachion, la savane arborée à Pterocarpus erinaceus et Hyptis suaveolens, la savane boisée à Daniellia oliveri et Chromolaena odorata, la savane boisée à Isoberlinia doka et Pennisetum polystachion, la savane arborée à Daniellia oliveri et Andropogon tectorum et la savane arborée inondable à Daniellia oliveri et Leersia hexandra. Les paramètres floristiques et structuraux changent d'un groupement à l'autre. Ainsi, la richesse spécifique varie de 49 à 153 espèces et la diversité des familles est comprise entre 22 et 49 avec une prédominance des Leguminosae. La densité évolue de 95 à 275 tiges/ha, la surface terrière de 2,35 à $8,44 \mathrm{~m}^{2} / \mathrm{ha}$. Conclusion et application des résultats: L'étude a permis d'évaluer la diversité floristique de la réserve de Ouoghi. De plus, elle a indiqué la nécessité de sa protection intégrale pour sa dynamique vers une végétation de forêt dense sèche. Une gestion durable de ce site nécessite l'implication des populations riveraines.

Mots-clés : Phytodiversité, groupements végétaux, végétation, zone soudano-guinéenne

\section{1- Introduction}

Les forêts tropicales de l'Afrique de l'Ouest constituent l'un des écosystèmes les plus diversifiés de la planète, avec un taux d'endémisme élevé (Myers et al., 2000). Beaucoup de choses y restent à étudier, cependant 
elles subissent une exploitation anthropique très forte et perdent chaque année 0,5 à $1 \%$ de leur superficie (Achard et al., 2002). Les taux les plus alarmants de destruction des milieux naturels sont enregistrés dans les pays comme le Togo $(1,44 \%)$, le Ghana $(1,26 \%)$, le Bénin $(1,25 \%)$, la Guinée $(1,12 \%)$ et le Nigeria $(0,86 \%)$. L'agriculture itinérante sur brûlis est la principale cause de la déforestation dans ces pays (FAO, 2005).

Au Bénin, 70.000 hectares de forêts sont dégradés en moyenne par an (FAO, 2010) et la pression anthropique s'accroît de jour en jour. En effet, face à la croissance démographique galopante, les besoins en bois d'énergie et de services et du pâturage sont considérables. Les aires protégées et parcs nationaux qui étaient de 2.158 .026 ha, soit 19,16\% de la superficie du Bénin, ont connu une réduction sensible en termes de superficie (DGRF, 2009). Pourtant, FAO (2010) a souligné que la dégradation des écosystèmes constitue une menace pour la biodiversité, car la destruction des milieux naturels conduit inévitablement à la rareté, voire à la disparition des espèces. Pour éviter ou amoindrir les conséquences catastrophiques qui découleraient de la poursuite de la destruction des milieux naturels, l'Etat béninois a mis en œuvre des programmes de plantations et de reboisement des écosystèmes naturels. Ce qui devrait permettre de freiner la dégradation des ressources naturelles et de conserver le patrimoine forestier national. C'est dans ce cadre que 15.000 ha de teckeraie, soit $0,13 \%$ de la superficie du pays, ont été mis en place (Ganglo, 1999). Aussi, la loi 93 - 009 du 2 juillet 1993 portant régime des forêts du Bénin a-t-elle été adoptée pour réglementer l'exploitation forestière en République du Bénin. Cependant, le couvert végétal national continue toujours de subir la réduction de sa superficie.

La problématique actuelle au Bénin est l'insuffisance et la non diversification des recherches d'accompagnement qui doivent guider les plans d'aménagement du territoire pour un renforcement des capacités locales dans un contexte de gestion participative afin de garantir la conservation et l'utilisation durable de la biodiversité. Les travaux du genre n'ont pour la plupart porté que sur les aires protégées et les parcs nationaux (Heymans et Petit, 1985 ; Sokpon, 1995 ; Trekpo, 1999 ; Amadou Siako, 2003). Par contre, les forêts privées ou forêts villageoises et les forêts sacrées, non régies par une loi étatique, sont peu prises en compte (Agbo et Sokpon, 1997 ; Amahowé, 1998 ; APROFOND-BÉNIN, 2000 ; Sego, 2008). Pourtant, elles jouent aussi le rôle de réserve naturelle. La présente étude a pour objectif d'inventorier les espèces végétales et de caractériser la végétation de la réserve forestière de Ouoghi dans la Commune de Savè. 


\section{Matériel et méthode}

\subsection{Milieu d'étude}

Le milieu d'étude est situé dans l'arrondissement de Sakin dans la commune de Savè. Il s'étend entre les parallèles $8^{\circ} 01^{\prime} 20^{\prime \prime}$ et $8^{\circ} 10^{\prime} 17^{\prime}$ ' de latitude Nord et les méridiens $2^{\circ} 28^{\prime} 41^{\prime \prime}$ et $2^{\circ} 39^{\prime} 02^{\prime \prime}$ de longitude Est (Figure 1 ).

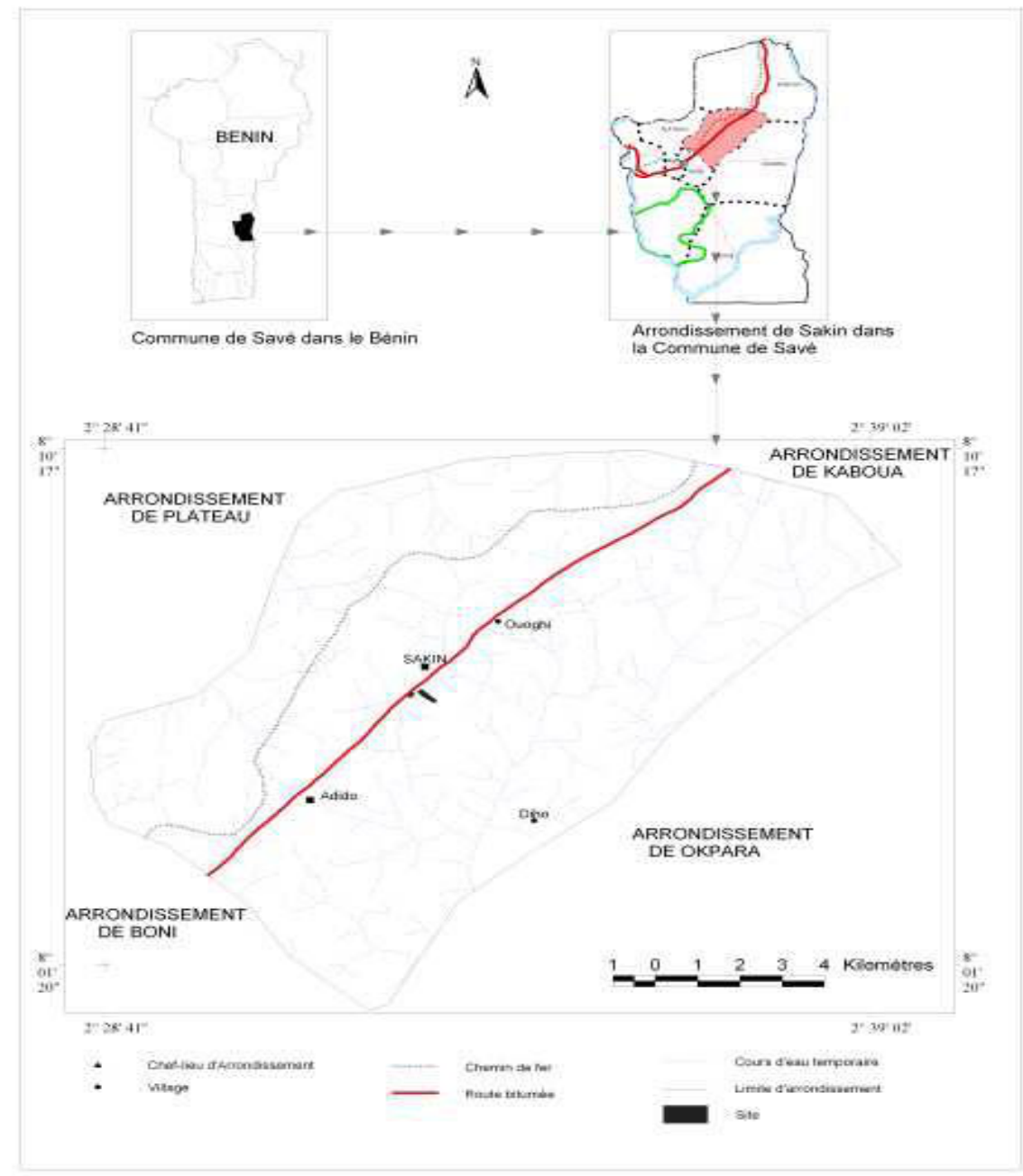

Figure 1: Localisation géographique du site d'étude dans l'arrondissement de Sakin.

Le climat est de type tropical humide de transition, caractérisé tantôt par quatre saisons (deux pluvieuses et deux sèches), tantôt par deux saisons (une pluvieuse et une sèche) (Akoègninou, 2004) avec une température moyenne de $27,67{ }^{\circ} \mathrm{C}$ et des précipitations moyennes annuelles de 1107,99 $\mathrm{mm}$ avec 5 mois de période sèche. La végétation est une mosaïque de savanes, de forêts claires parsemées de galeries forestières, des champs et 
des plantations (Akoègninou et al., 2006). Les sols sont de types ferrugineux tropicaux (Faure, 1977 ; Dubroeucq, 1977).

La population de Savè qui était de 67753 habitants en 2002 est passée à 87177 habitants en 2013, soit un taux d'accroissement annuel de 2,86\% (INSAE, 2015).

La principale activité de la population est l'agriculture sur brûlis. Les activités secondaires sont la chasse, l'élevage, l'exploitation forestière et le tourisme.

\subsection{Méthode d'étude}

\subsubsection{Collecte des données}

Le site d'étude est composé de deux blocs dont l'un a une superficie de 6,54 ha et l'autre 1,39 ha, soit au total 7,93 ha. Des placeaux rectangulaires de $25 \mathrm{~m} \times 20 \mathrm{~m}$ (soit $500 \mathrm{~m}^{2}$ ) ont été installés de façon contiguë sur chacun des deux blocs (Figure 2). Au total, 144 placeaux ont été installés dont 120 sur le grand bloc et 24 sur le petit bloc. La superficie inventoriée est alors de 7,20 ha, soit un taux de sondage de 90,79\%.

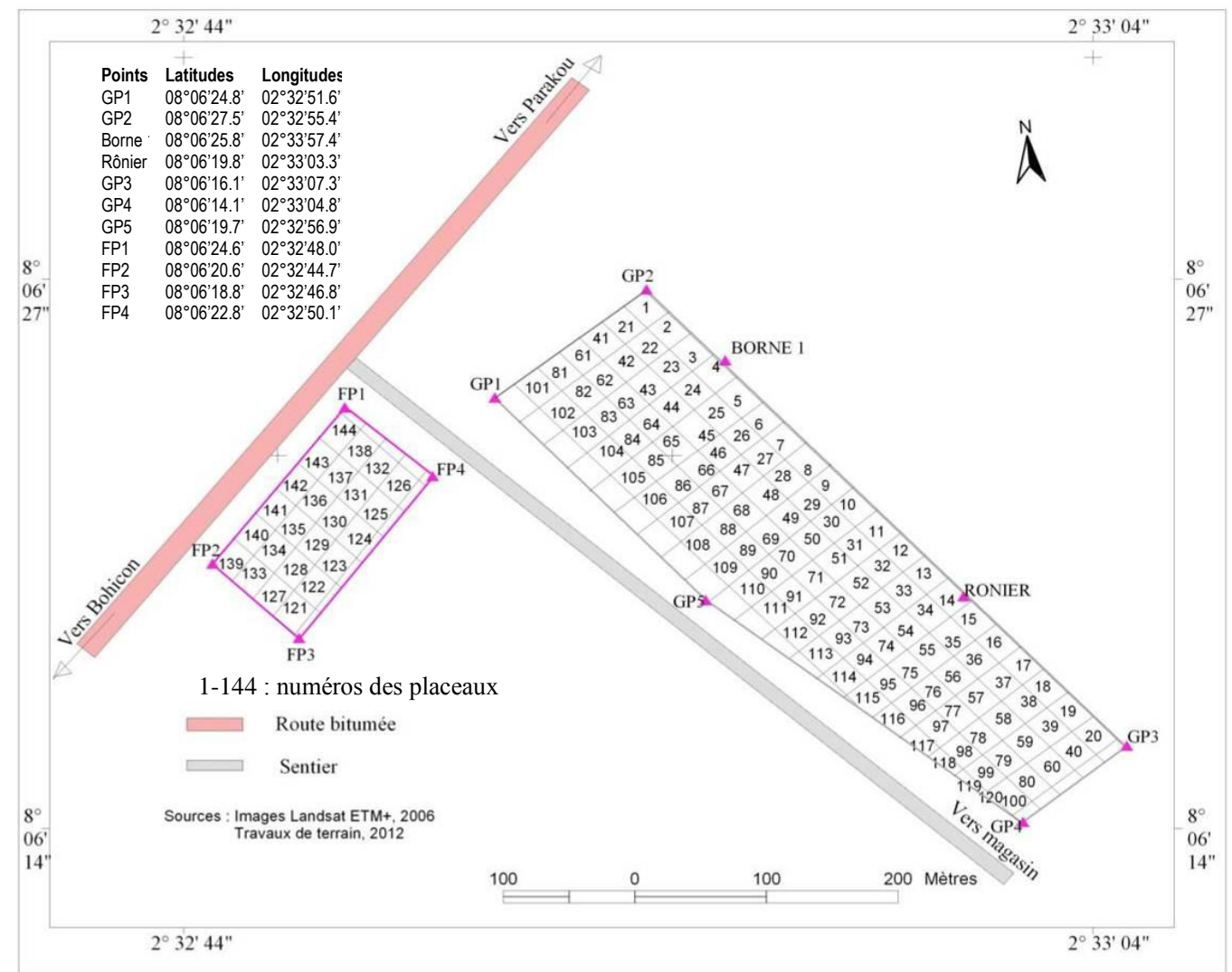

Figure 2 : Répartition des placeaux de relevés floristiques du site 
Les relevés phytosociologiques ont été réalisés suivant la méthode de BraunBlanquet (1932). Les espèces ont été enregistrées par strate avec leur coefficient d'abondance-dominance. Le coefficient d'abondance-dominance d'une espèce exprime le nombre d'individus et son recouvrement par unité de surface dans le milieu. Il varie de 0 à 5 :

$+:$ individus rares ou très rares et à recouvrement très faible $(<1 \%)$;

$1:$ individus assez abondants, mais à recouvrement faible ( 1 à $5 \%)$;

2 : individus très abondants et à recouvrement compris entre 6 et $25 \%$

;

3 : nombre d'individus quelconque, recouvrement allant de 26 à $50 \%$

4 : nombre d'individus quelconque, recouvrement allant de 51 à $75 \%$ à $100 \%$.

$5:$ nombre d'individus quelconque et recouvrement compris entre 76

Pour les mesures dendrométriques, les diamètres à hauteur de poitrine d'homme (d.b.h.), soit à $1,30 \mathrm{~m}$ au-dessus du sol ou à $30 \mathrm{~cm}$ au-dessus des contreforts ont été enregistrés pour tous les individus de d.b.h. $\geq 10 \mathrm{~cm}$.

La plupart des espèces ont été directement identifiées sur le terrain avec la Flore Analytique du Bénin (Akoègninou et al., 2006) et Arbres et arbustes du Sahel (Arbonnier, 2009). Le reste des échantillons a été identifié par comparaison avec les spécimens de référence de l'Herbier National du Bénin.

\subsubsection{Traitement des données}

La matrice des 144 relevés et 246 espèces a été constituée en présence-absence. Elle a été soumise à l'ordination qui est la " Detrented Correspondence Analysis (DECORANA ou DCA) ». La matrice issue de chacun des 2 groupes de relevés individualisés par la DCA a été soumise à la classification. Elle a permis d'identifier les groupes de relevés ayant des compositions floristiques semblables. Ces groupes de relevés sont assimilés à des formations végétales ou des groupements végétaux selon le cas. Le logiciel utilisé est le Community Analysis Package (CAP) (Hendeson et Seaby, 2002).

La diversité floristique est évaluée à l'aide de la richesse spécifique, de la diversité en genre et en familles (Daget 1980). La moyenne par groupement de la diversité spécifique a été déterminée avec son écart-type pour les différents groupements, étant donné que le nombre de relevés varie d'un groupement à un autre. La nomenclature botanique utilisée est celle de Akoègninou et al. (2006).

Le spectre biologique brut est établi à partir des types biologiques définis par Raunkiaer (1934). 
Le spectre biogéographique est réalisé à partir des types biogéographiques selon White (1983).

Les différentes communautés végétales ont été caractérisées sur la base de leur indice phytogéographique $\left(\mathrm{I}_{\mathrm{p}}\right)$ (Adomou et al., 2007) : $\mathrm{I}_{\mathrm{p}}=(\mathrm{S}+$ $\mathrm{SZ} / \mathrm{GC}$, avec S: le nombre d'espèces soudaniennes, SZ: le nombre d'espèces soudano-zambéziennes et GC: le nombre d'espèces guinéocongolaises. Lorsque $\mathrm{I}_{\mathrm{p}}<1$, la flore humide est dominante, par contre la flore sèche est dominante si $\mathrm{I}_{\mathrm{p}}>1$.

Les paramètres structuraux ont été analysés à l'aide de : la surface terrière, la densité et l'indice de diversité de Shannon.

La densité des peuplements $(\mathrm{N})$ est le nombre de tiges par hectare. Elle a été évaluée pour les arbres et arbustes à d.b.h. $\geq 10 \mathrm{~cm}$.

La surface terrière du peuplement est la somme des surfaces terrières de tous les arbres et arbustes à d.b.h. $\geq 10 \mathrm{~cm}$ et qui constituent le peuplement. Elle a été obtenue par la formule :

$\mathrm{G}=\sum \pi \mathrm{D}^{2} / 4$, avec $\mathrm{G}=$ surface terrière exprimée en $\mathrm{m}^{2} /$ ha et $\mathrm{D}=$ diamètre à hauteur de poitrine d'homme des arbres.

L'indice de diversité de Shannon a permis d'évaluer la diversité des ligneux de d.b.h. $\geq 10 \mathrm{~cm}$. Il est donnée par la formule: $\mathrm{H}=$ $\Sigma(\mathrm{Ni} / \mathrm{N}) \log 2(\mathrm{Ni} / \mathrm{N})$, avec $\mathrm{Ni}=$ l'effectif de l'espèce $\mathrm{i}$ et $\mathrm{N}$, l'effectif total des espèces (Legendre et Legendre 1984; Pichod-Viale 1991, 1995). La diversité est faible lorsque $\mathrm{H}<3$ bits, moyenne si $\mathrm{H}$ est compris entre 3 et 4 puis élevée quand $\mathrm{H} \geq 4$ bits.

3. Résultats

\subsection{Typologique des formations végétales du site d'étude}

\subsubsection{Ordination des relevés floristiques du site d'étude}

La matrice des 144 relevés et 246 espèces, soumise à la Detrended Correspondence

Analysis (DCA), discrimine 2 groupes de relevés correspondant aux 2 blocs de relevés

(Figure 3) :

- le premier comprend les 120 relevés effectués sur le grand bloc (GP), où le sol est peu profond et renferme des concrétions latéritiques superficielles ;

- le second groupe réunit les 24 relevés exécutés sur le petit bloc (PP) qui est situé sur

un sol plus profond que le précédent et sans concrétions latéritiques superficielles. L'axe 1 porte vers son origine les 120 relevés du GP et à son extrémité droite, les relevés du PP. Il traduit un gradient structural du sol. 
L'indice de similarité de Jaccard entre les deux groupes de relevés est de $44,72 \%$. Le taux de dissemblance entre eux est alors de 55,28\% et indique que les deux blocs sont

floristiquement distincts.

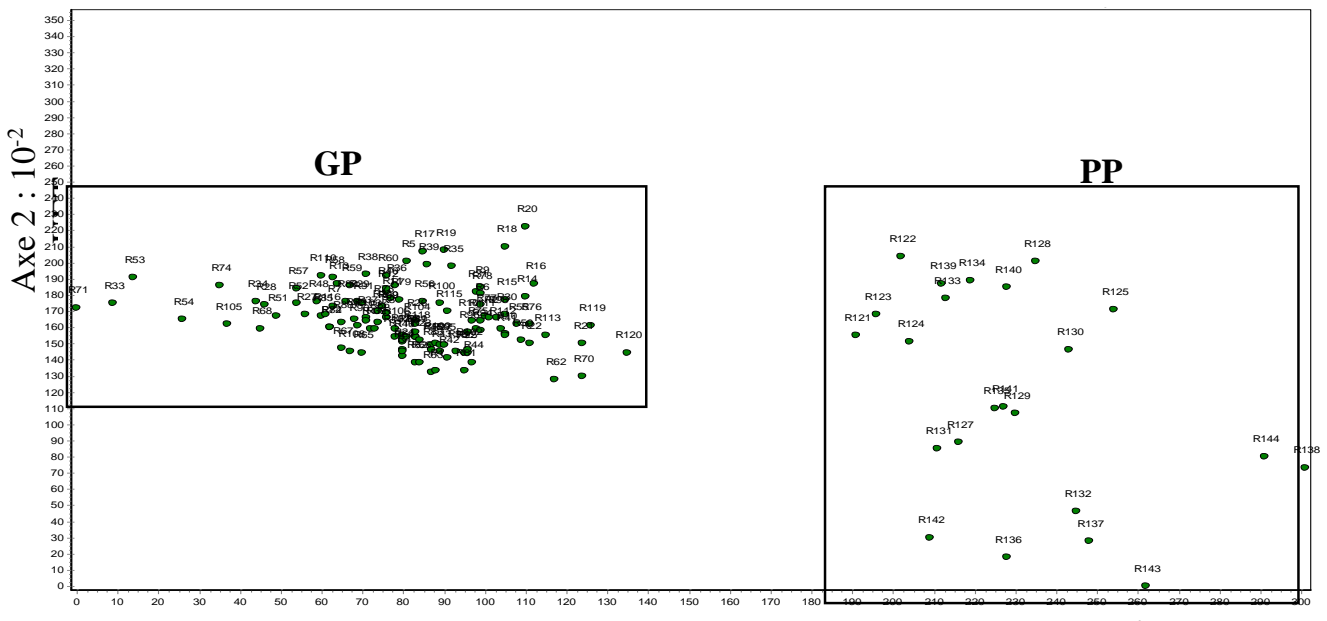

Axe $1: 10^{-2}$ UET

Figure 3 : DCA des relevés floristiques des deux blocs

GP : groupe des relevés du grand bloc ; PP : groupe des relevés du petit bloc ; UET : Unité d'Ecart-Type ; Axe 1 (score : 0,30 ; longueur de gradient : 3,50 UET) ; Axe 2 (score : 0,16; longueur de gradient : 2,41 UET).

La classification hiérarchique ascendante appliquée à la matrice des 120 relevés et 217 espèces végétales du grand bloc (GP) discrimine, à $51 \%$ de dissemblance, trois formations végétales subdivisées en 6 groupes végétaux (Figure 4). La savane arborée est composée de trois groupements végétaux (désignés par $\mathrm{A}, \mathrm{B}$ et $\mathrm{C}$ ), la savane boisée est constituée de deux groupements végétaux ( $\mathrm{D}$ et $\mathrm{E}$ ) et la savane arbustive ne comporte qu'un seul groupement végétal $(\mathrm{F})$. 


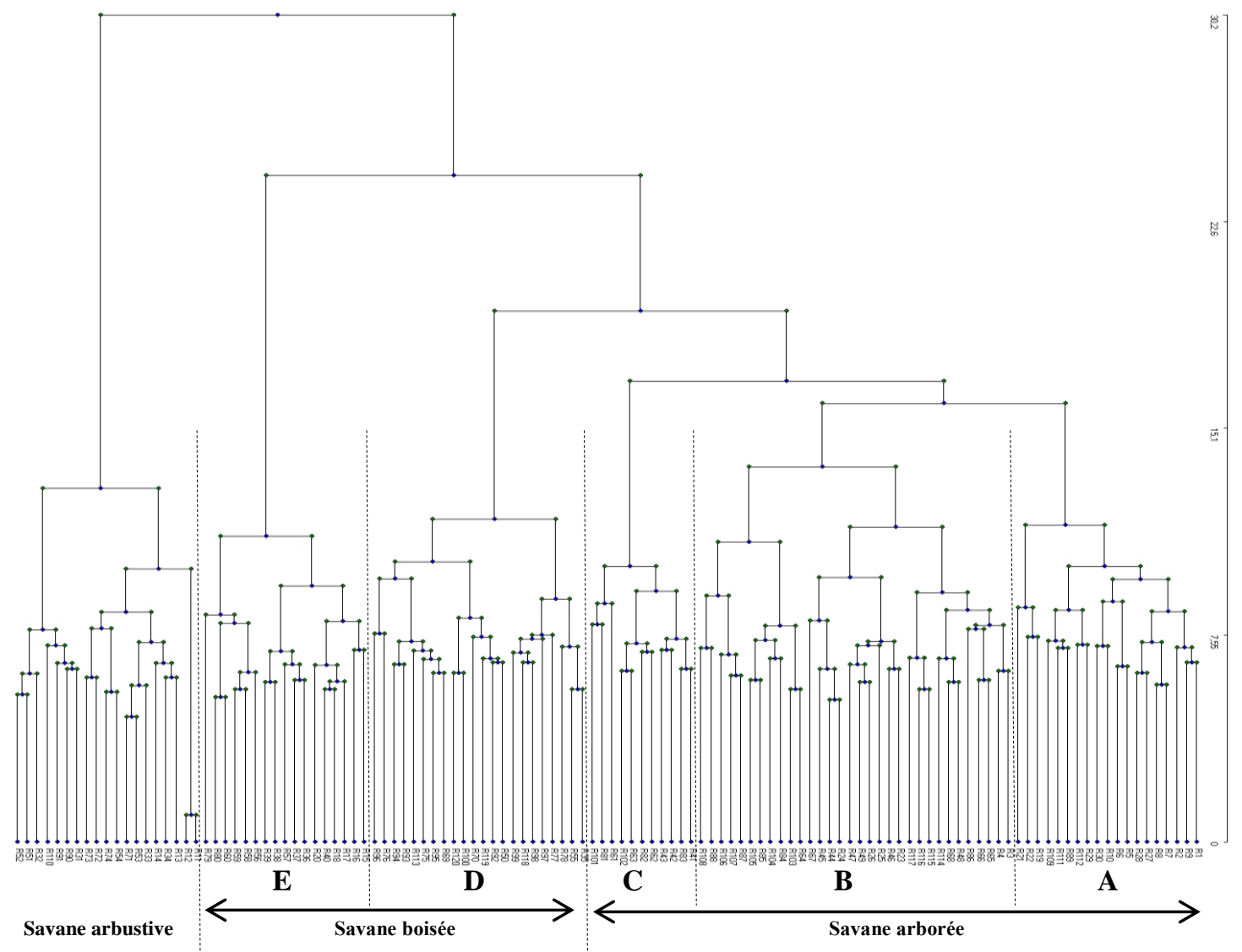

Figure 4 : Dendrogramme des relevés du grand bloc

A - C : savane arborée (A : groupement à Hymenocardia acida et Pennisetum polystachion,

$\mathrm{B}$ : groupement à Vitellaria paradoxa et Pennisetum polystachion, $\mathrm{C}:$ groupement à Pterocarpus erinaceus et Hyptis suaveolens); D et E : savane boisée (D : groupement à Daniellia oliveri et Chromolaena odorata, E : groupement à Isoberlinia doka et Pennisetum polystachion) ; F : savane arbustive (F : groupement à Prosopis africana et Pennisetum polystachion)

La figure 5 présente la classification hiérarchique ascendante de la matrice des 24 relevés et 138 espèces végétales du petit bloc (PP). Elle individualise 2 groupements végétaux de savane arborée. 


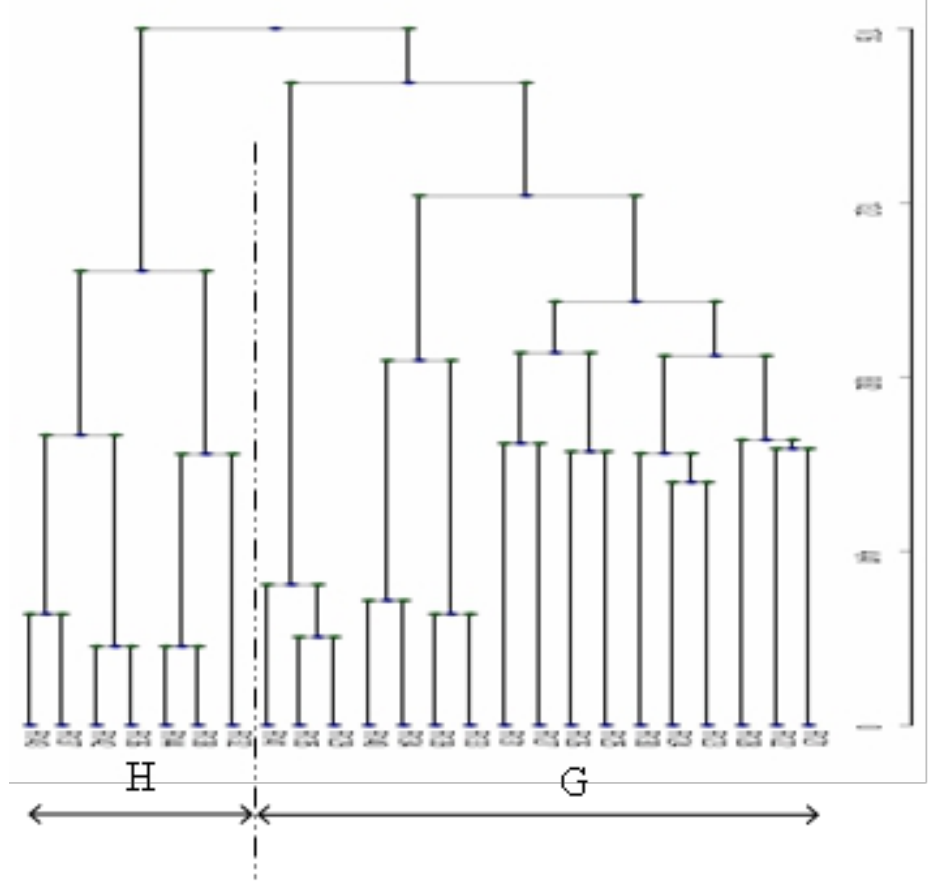

Figure 5 : Dendrogramme des relevés du petit bloc

$\mathrm{G}$ et $\mathrm{H}$ : savane arborée (G: groupement à Daniellia oliveri et Andropogon tectorum et $\mathrm{H}$ : groupement à Daniellia oliveri et Leersia hexandra).

Le tableau 1 montre les principales espèces de chacun des groupements végétaux avec leur coefficient moyen d'abondance-dominance et leur classe de fréquence. 
Tableau 1: Groupements végétaux et leurs principales espèces.

Pour les abréviations A-H, voir les figures 4 et 5 . Les nombres en chiffres romains indiquent la classe de fréquence (F) des espèces dans le groupement correspondant (I : $\leq$ II : 20-40\%, III : 40-60\%, IV : 60-80\%, V : 80-100\%). Ab-dom : coefficient moyen d'abondance-dominance.

\begin{tabular}{|c|c|c|c|c|c|c|c|c|c|c|c|c|c|c|c|c|}
\hline Groupements végétaux & $\mathrm{A}$ & & $\mathrm{B}$ & & $\mathrm{C}$ & & $\mathrm{D}$ & & $\mathrm{E}$ & & $\mathrm{F}$ & & $\mathrm{G}$ & & $\mathrm{H}$ & \\
\hline Espèces & $\mathrm{F}$ & $\begin{array}{l}\text { Ab- } \\
\text { dom }\end{array}$ & $\mathrm{F}$ & $\begin{array}{l}\text { Ab- } \\
\text { dom }\end{array}$ & $\mathrm{F}$ & $\begin{array}{l}\text { Ab- } \\
\text { dom }\end{array}$ & $\mathrm{F}$ & $\begin{array}{l}\text { Ab- } \\
\text { dom }\end{array}$ & $\mathrm{F}$ & $\begin{array}{l}\text { Ab- } \\
\text { dom }\end{array}$ & $\mathrm{F}$ & $\begin{array}{l}\begin{array}{l}\text { Ab- } \\
\text { dom }\end{array} \\
\end{array}$ & $\mathrm{F}$ & $\begin{array}{l}\text { Ab- } \\
\text { dom }\end{array}$ & $\mathrm{F}$ & $\begin{array}{l}\text { Ab- } \\
\text { dom }\end{array}$ \\
\hline Acacia sieberiana DC. Var .villosa & & & & & & & II & 1,1 & & & & & & & & \\
\hline Acroceras zizanioides (Kunth) Dandy & & & & & & & & & & & & & & & II & 2 \\
\hline Afzelia africana $\mathrm{Sm}$ & & & & & & & & & I & 1,5 & & & & & & \\
\hline Amorphophallus dracontioides (Engl.) N.E.Br & & & & & & & & & I & 1,5 & & & & & & \\
\hline Ampelocissus leonensis (Hook.f.) Planch & & & & & & & I & 2 & & & & & & & & \\
\hline Anchomanes difformis (Blume) Engl & & & & & & & & & & & & & & & III & 1,6 \\
\hline Andropogon gayanus Kunth var. gayanus, DC & & & & & & & I & 2 & & & & & & & & \\
\hline Andropogon tectorum Schumach. \& Thonn & & & & & & & & & & & & & $\mathrm{V}$ & 1,7 & $\mathrm{~V}$ & 2,2 \\
\hline $\begin{array}{l}\text { Annona senegalensis Pers. Ssp. Oulotricha Le Thomas } \\
\text { ex Le Thomas } \\
\text { Anogeissus leiocarpa (DC) Guill. \& Perr }\end{array}$ & $\mathrm{V}$ & 1,3 & $\mathrm{~V}$ & 1,4 & V & $\begin{array}{l}1,2 \\
1,2\end{array}$ & $\mathrm{~V}$ & 1,3 & $\mathrm{~V}$ & 1,1 & & & IV & 1,1 & & \\
\hline Azadirachta indica A. Juss & $\mathrm{V}$ & 1,2 & IV & 1,2 & $\mathrm{~V}$ & 1,8 & $\mathrm{~V}$ & 1,2 & & & III & 1,4 & & & III & 1,3 \\
\hline Basilicum polystachyon (L .) Moench & IV & 1,3 & $\mathrm{~V}$ & 1,2 & $\mathrm{~V}$ & 1,4 & IV & 1,4 & $\mathrm{~V}$ & 1,2 & II & 1,1 & & & & \\
\hline Biophytum petersianum Klotzch & $\mathrm{V}$ & 1,3 & $\mathrm{~V}$ & 1,2 & $\mathrm{~V}$ & 1,1 & & & $\mathrm{~V}$ & 1,1 & & & & & & \\
\hline Borassus aethiopum Mart & II & 1,2 & IV & 1,5 & II & 1,5 & II & 1,4 & & & I & 2 & II & 1,5 & $\mathrm{~V}$ & 1,1 \\
\hline Brachiaria deflexa (Schumach.) Robyns & & & & & & & & & & & & & II & 1,3 & IV & 1,4 \\
\hline Brachiaria jubata (Fig. \& De Not.) Stapf & & & & & & & & & & & & & I & 2 & & \\
\hline Bridelia ferruginea Benth & $\mathrm{V}$ & 1,2 & II & 1,6 & & & IV & 1,2 & IV & 1,1 & & & IV & 1,2 & & \\
\hline Bulbostylis hensii (C.B.Clarke) R.W.Haines & I & 1,3 & & & & & & & & & I & 1,3 & & & & \\
\hline Burkea africana Hook & IV & 1,7 & II & 1,1 & & & III & 1,3 & IV & 1,4 & & & III & 1,5 & & \\
\hline Chamaecrista rotundifolia (Pers.) Greene & & & & & & & & & & & II & 1,1 & & & & \\
\hline Chromolaena odorata (L.) R.M.King & IV & 1,2 & & & III & 1,1 & III & 1,8 & $\mathrm{~V}$ & 1,3 & & & IV & 1,6 & III & 2,6 \\
\hline Cissus petiolata Hook.f. & & & & & & & & & & & & & I & 2 & & \\
\hline
\end{tabular}




\begin{tabular}{|c|c|c|c|c|c|c|c|c|c|c|c|c|c|c|c|c|}
\hline $\begin{array}{l}\text { Cissus populnea Guill. \& Perr } \\
\text { Combretum molle R.Br. Ex G.Don } \\
\text { Cussonia arborea Hochst. ex A. Rich } \\
\text { Cyphostemma adenocaule (Steud.) Desc }\end{array}$ & II & 1,2 & IV & $\begin{array}{l}1,1 \\
1,4\end{array}$ & $\mathrm{~V}$ & 1,4 & III & 1,1 & & & & & I & 2 & & \\
\hline Daniellia oliveri (Rolfe) Hutch. \& Dalziel & $\mathrm{V}$ & 1,8 & $\mathrm{~V}$ & 1,6 & III & 1,4 & $\mathrm{~V}$ & 2,7 & $\mathrm{~V}$ & 1,7 & $\mathrm{~V}$ & 1,4 & $\mathrm{~V}$ & 2 & $\mathrm{~V}$ & 1,7 \\
\hline Digitaria horizontalis Willd. & II & 1,8 & II & 1,1 & & & II & 1,2 & II & 1,6 & & & & & & \\
\hline Dioscorea togoense Knuth & II & 1,3 & & & & & & & & & & & & & & \\
\hline Diospyros mespiliformis Hochst. ex A. DC. & & & II & 1,1 & & & & & & & & & & & & \\
\hline Dolichos argenteus Willd. & & & & & & & & & & & I & 1,3 & & & & \\
\hline Entada africana Guill. \& Perr & & & II & 1,2 & & & III & 1,1 & & & & & & & & \\
\hline Eragrostis aspera (Jacq.) Nees & & & I & 1,3 & & & & & & & & & & & & \\
\hline Eriosema glomeratum (Guill. \& Perr.) Hook. f. & II & 1,2 & III & 1,2 & & & & & & & & & & & & \\
\hline Eriosema psoraleoides (Lam.) G.Don & & & & & & & & & & & & & III & 1,1 & & \\
\hline Ficus sycomorus L. & I & 2,0 & & & II & 2 & & & & & I & 2 & & & & \\
\hline Ficus sur Forssk. & II & 1,5 & & & & & II & 1,1 & & & II & 1,2 & III & 1,5 & & \\
\hline Flueggea virosa (Roxb. ex Willd.) Voigt & $\mathrm{V}$ & 1,1 & $\mathrm{~V}$ & 1,2 & & & $\mathrm{~V}$ & 1,1 & $\mathrm{~V}$ & 1,1 & IV & 1,2 & & & & \\
\hline Gardenia erubescens Stapf \& Hutch. & II & 1,1 & & & & & & & II & 1,5 & & & & & & \\
\hline Gymnosporia senegalensis (Lam.) Loes. & & & III & 1,2 & & & & & & & & & & & & \\
\hline Haumaniastrum buettneri (Gürke) J.K.Morton & & & I & 1,2 & & & & & & & & & & & & \\
\hline Hymenocardia acida Tul. & $\mathrm{V}$ & 1,6 & $\mathrm{~V}$ & 1,4 & $\mathrm{~V}$ & 1,6 & $\mathrm{~V}$ & 1,4 & $\mathrm{~V}$ & 1,6 & & & $\mathrm{~V}$ & 1,5 & & \\
\hline Hyparrhenia smithiana (Hook. f.) Stapf & & & & & & & & & I & 2 & & & $\mathrm{~V}$ & 1,5 & & \\
\hline Hyptis suaveolens (L.) Poit & $\mathrm{V}$ & 1,9 & $\mathrm{~V}$ & 1,2 & $\mathrm{~V}$ & 1,9 & II & 1,1 & II & 1,3 & IV & 1,7 & & & & \\
\hline Imperata cylindrica (L.) P. Beauv. & $\mathrm{V}$ & 1,3 & $\mathrm{~V}$ & 1,3 & & & IV & 1,4 & IV & 1,1 & IV & 1,4 & III & 1,1 & & \\
\hline Indigofera hirsuta $\mathrm{L}$. & II & 1,3 & III & 1,1 & II & 1,6 & II & 1,1 & & & & & & & & \\
\hline Isoberlinia doka Craib \& Stapf & & & & & & & & & $\mathrm{V}$ & 2,1 & & & & & & \\
\hline
\end{tabular}




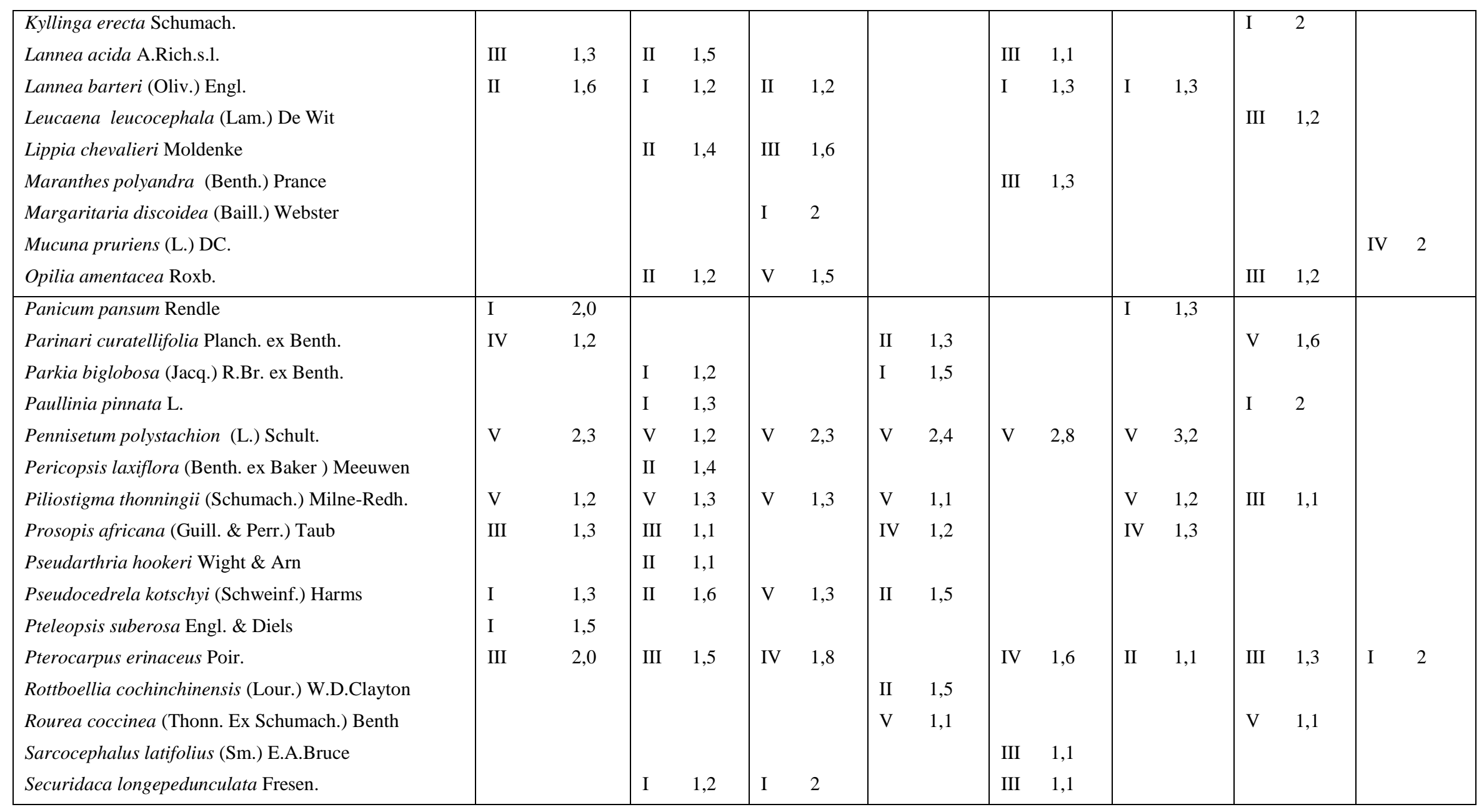




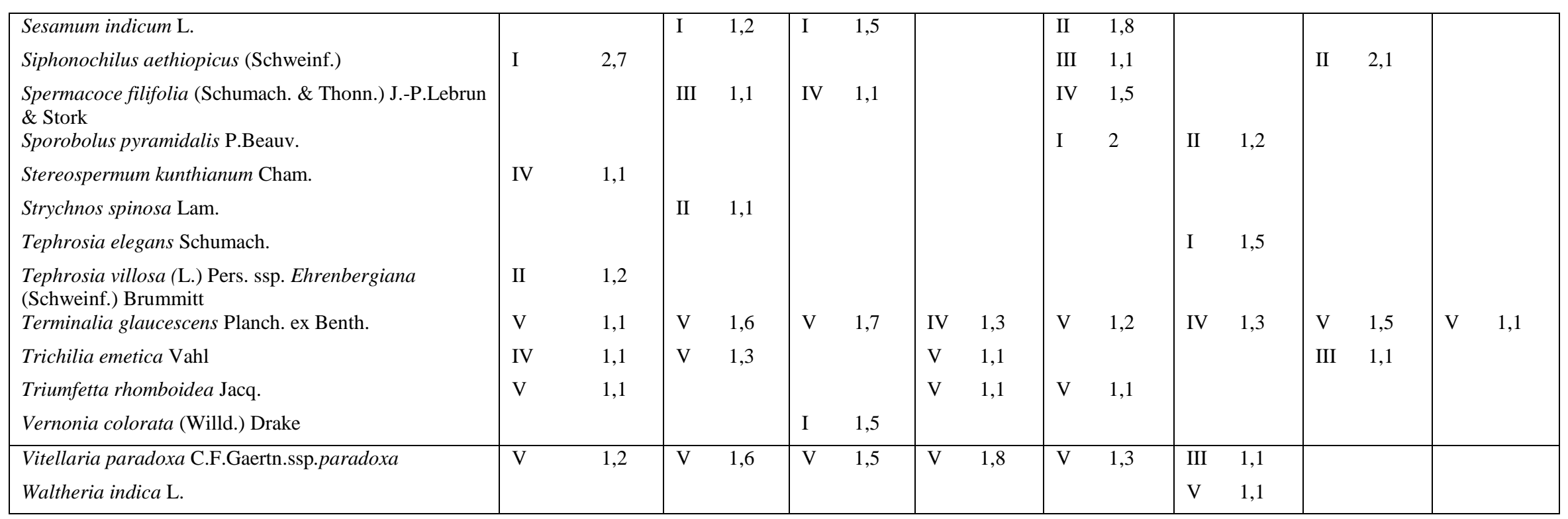




\subsection{Caractéristiques floristiques du site}

\subsubsection{Diversité floristique}

La flore du site de Ouoghi compte 246 espèces appartenant à 178 genres et 63 familles. Le tableau 2 présente la diversité floristique de chacun des groupements. Les valeurs moyennes par groupement de la diversité spécifique, de la diversité en genre et en familles varient d'un groupement à un autre. Le groupement à Vitellaria paradoxa et Pennisetum polystachion est le plus diversifié en espèces (153) et en genres (126), alors que le groupement à Daniellia oliveri et Chromolaena odorata est le riche en famille (49). Les Leguminosae constituent la famille la plus riche en espèces au niveau de tous les groupements.

\subsubsection{Spectre biologique}

La figure 6 présente les spectres biologiques des huit groupements végétaux obtenus sur les deux blocs qui constituent le site. Les phanérophytes sont prédominantes $(45,97$ à $67,35 \%)$ au niveau des différents groupements végétaux du site. Les parasites sont représentés uniquement au niveau du groupement à Pterocarpus erinaceus et Hyptis suaveolens situé sur le grand bloc du site.

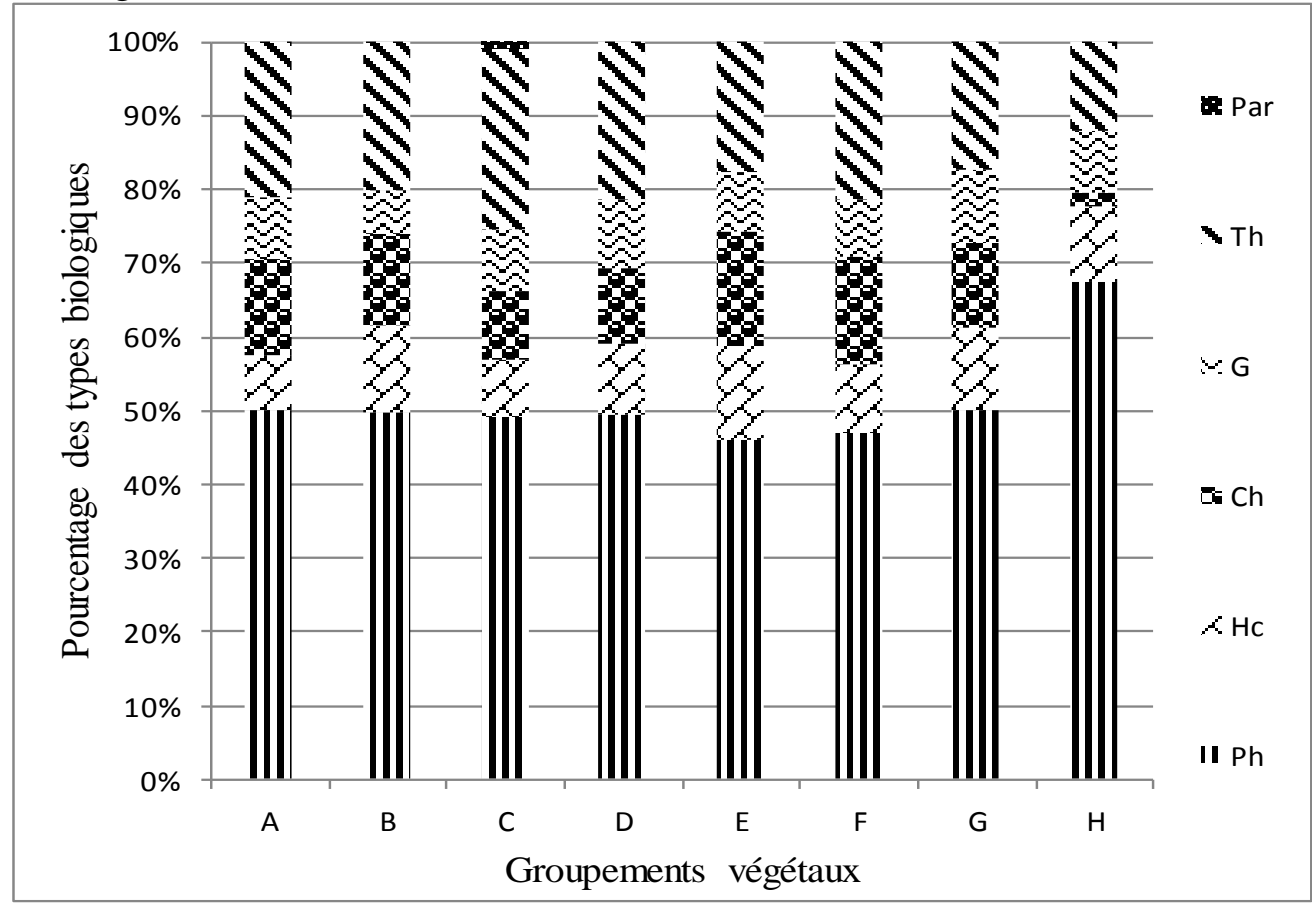

Figure 6 : Spectre biologique des groupements végétaux.

$\mathrm{Ph}$ : phanérophytes, Hc : hémicryptophytes, Ch : Chaméphytes, G : géophytes, Th :

Thérophytes, Par : Parasites. Pour les abréviations A à H, voir les figures 4 et 5 . 
Tableau 2 : Diversité floristique des groupements végétaux

Pour les abréviations $\mathrm{A}$ à $\mathrm{H}$, voir les figures 4 et 5 . Fv : formation végétale, $\mathrm{Gv}$ : groupement végétal, Rt : richesse spécifique totale, $\mathrm{Rm}$ : richesse spécifique moyenne, Er : écart-type de

$\mathrm{Rm}, \mathrm{Ng}$ : nombre de genres, $\mathrm{Nf}$ : nombre de familles.

\begin{tabular}{|c|c|c|c|c|c|c|}
\hline Site & $\mathrm{Fv}$ & Gv & $\begin{array}{c}\mathrm{Rt} \\
(\mathrm{Rm} \pm \mathrm{Er})\end{array}$ & $\mathrm{Ng}$ & $\mathrm{Nf}$ & Familles dominantes \\
\hline \multirow{6}{*}{ 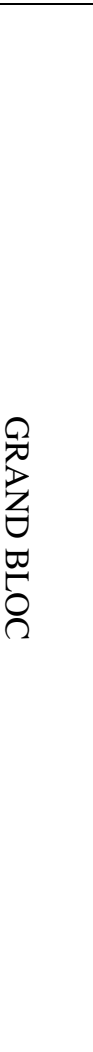 } & \multirow{3}{*}{ 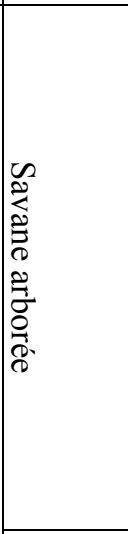 } & A & $\begin{array}{c}145 \\
(42,73 \pm 4,09)\end{array}$ & 118 & 45 & $\begin{array}{c}\text { Leguminosae }(22,06 \%), \\
\text { Asteraceae }(7,58 \%), \text { Poaceae et } \\
\text { Rubiaceae }(6,89 \%), \\
\text { Euphorbiaceae }(4,82 \%) .\end{array}$ \\
\hline & & B & $\begin{array}{c}153 \\
(46,62 \pm 6,19) \\
\end{array}$ & 126 & 48 & $\begin{array}{c}\text { Leguminosae }(22,22 \%), \\
\text { Asteraceae }(7,84 \%), \text { Poaceae } \\
(7,18 \%), \text { Euphorbiaceae et } \\
\text { Rubiaceae }(5,88 \% \text { chacune }) .\end{array}$ \\
\hline & & $\mathrm{C}$ & $\begin{array}{c}118 \\
(46,45 \pm 6,37)\end{array}$ & 98 & 41 & $\begin{array}{c}\text { Leguminosae }(16,94 \%), \\
\text { Asteraceae }(9,32 \%), \\
\text { Euphorbiaceae }(7,62 \%), \\
\text { Poaceae et Rubiaceae }(5,93 \% \\
\text { chacune). }\end{array}$ \\
\hline & \multirow{2}{*}{ 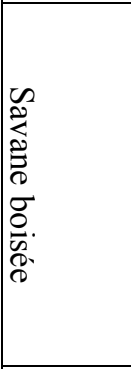 } & D & $\begin{array}{c}144 \\
(45,18 \pm 8,34)\end{array}$ & 119 & 49 & $\begin{array}{c}\text { Leguminosae }(22,22 \%), \\
\text { Asteraceae }(7,63 \%), \text { Poaceae } \\
(6,94 \%), \text { Euphorbiaceae et } \\
\text { Rubiaceae (4,86\% chacune). }\end{array}$ \\
\hline & & $\mathrm{E}$ & $\begin{array}{c}123 \\
(44,11 \pm 4,89)\end{array}$ & 100 & 39 & $\begin{array}{c}\text { Leguminosae }(21,13 \%), \\
\text { Asteraceae }(9,75 \%), \\
\text { Euphorbiaceae }(8,13 \%), \\
\text { Cyperaceae }(6,5 \%), \text { Poaceae et } \\
\text { Rubiaceae }(5,69 \% \text { chacune). }\end{array}$ \\
\hline & 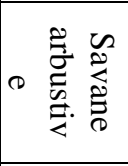 & $\mathrm{F}$ & $\begin{array}{c}116 \\
(29,47 \pm 6,49) \\
\end{array}$ & 93 & 41 & $\begin{array}{c}\text { Leguminosae }(23,27 \%) \text {, Poaceae } \\
\text { et Rubiaceae }(7,75 \% \text { chacune }), \\
\text { Asteraceae }(6,03 \%), \\
\text { Cyperaceae }(5,17 \%) .\end{array}$ \\
\hline \multirow{2}{*}{ 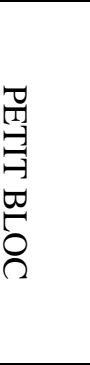 } & \multirow{2}{*}{ 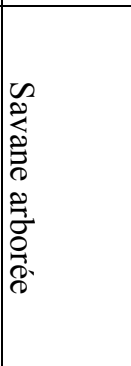 } & G & $\begin{array}{c}131 \\
(36,94 \pm 7,43)\end{array}$ & 101 & 41 & $\begin{array}{c}\text { Leguminosae }(24,24 \%), \\
\text { Poaceae, Asteraceae, } \\
\text { Euphorbiaceae et Rubiaceae } \\
(5,30 \% \text { chacune }) .\end{array}$ \\
\hline & & $\mathrm{H}$ & $\begin{array}{c}49 \\
(20,28 \pm 5,40)\end{array}$ & 47 & 22 & $\begin{array}{c}\text { Leguminosae }(18,36 \%) \text {, Poaceae } \\
(12,2 \%), \\
\text { Euphorbiaceae et Rubiaceae } \\
(8,16 \% \text { chacune }), \\
\text { Asteraceae }(6,12 \%) .\end{array}$ \\
\hline & \multicolumn{2}{|l|}{ Total } & $\begin{array}{c}246 \\
(40,7 \pm 9,6)\end{array}$ & 178 & 63 & $\begin{array}{c}\text { Leguminosae }(21,13 \%), \\
\text { Asteraceae et Poaceae }(8,53 \% \\
\text { chacune), Rubiaceae }(5,69 \%) \text {. }\end{array}$ \\
\hline
\end{tabular}

\subsubsection{Spectre phytogéographique}

La figure 7 montre les spectres phytogéographiques des différents groupements végétaux. Les espèces à larges distributions sont partout majoritaires $(34,84$ à $39,83 \%)$ à l'exception du groupement à Daniellia 
oliveri et Leersia hexandra de la savane arborée localisée sur le petit bloc du site où prédominent les espèces soudano-guinéennes.

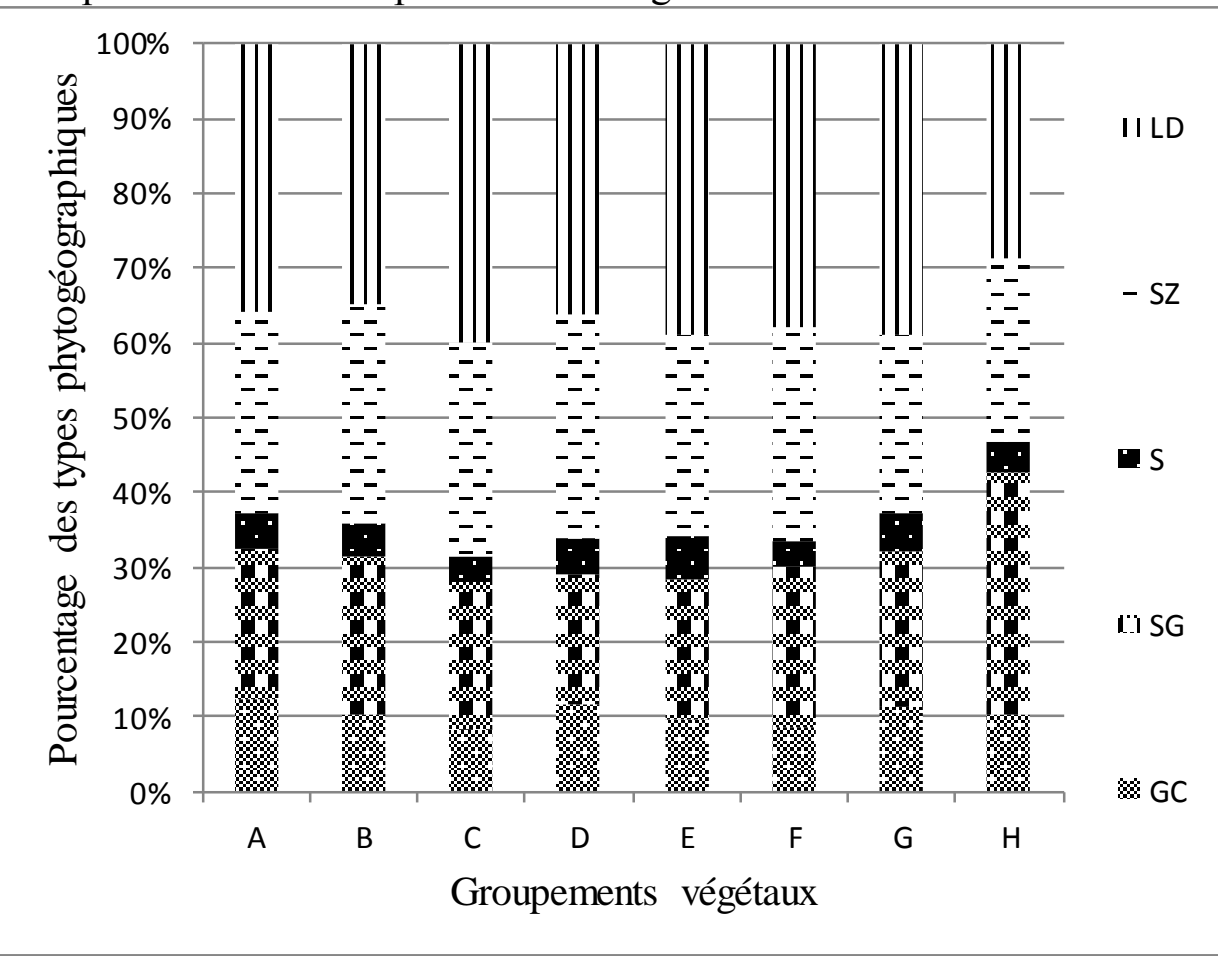

Figure 7 : Spectre phytogéographique des groupements végétaux.

Types phytogéographiques : GC : guinéo-congolais, SG : soudano-guinéen, S : soudanien, $\mathrm{SZ}$ : soudano-zambézien, $\mathrm{LD}$ : large distribution. Pour les abréviations $\mathrm{A}$ à $\mathrm{H}$, voir les figures 4 et 5 .

Les indices phytogéographiques des différents groupements sont consignés dans le tableau 4. Il ressort que les valeurs enregistrées varient de 4,4 à 9,81, elles indiquent que la flore sèche est abondante sur le site.

Tableau 3 : Indices phytogéographiques des groupements végétaux.

Pour les abréviations A à $\mathrm{H}$, voir les figures 4 et 5 . Fv : formation végétale, $\mathrm{Gv}$ : groupement végétal, Ip : Indice Phytogéographique.

\begin{tabular}{|c|c|c|c|}
\hline Site & $\mathrm{Fv}$ & $\mathrm{Gv}$ & Ip \\
\hline \multirow{6}{*}{ 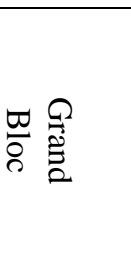 } & \multirow{3}{*}{ Savane arborée } & $\mathrm{A}$ & 9,17 \\
\hline & & $\mathrm{B}$ & 9,81 \\
\hline & & $\mathrm{C}$ & 7,4 \\
\hline & \multirow{2}{*}{$\begin{array}{l}\text { Savane } \\
\text { boisée }\end{array}$} & $\mathrm{D}$ & 9,53 \\
\hline & & $\mathrm{E}$ & 9,75 \\
\hline & Savane arbustive & $\mathrm{F}$ & 7,3 \\
\hline \multirow{2}{*}{ 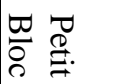 } & \multirow{2}{*}{ Savane arborée } & G & 9,07 \\
\hline & & $\mathrm{H}$ & $e_{1}$ \\
\hline
\end{tabular}




\subsubsection{Caractéristiques structurales des groupements ligneux}

La surface terrière, la densité et l'indice de diversité de Shannon des différents groupements ligneux sont consignés dans le tableau 4. La surface terrière moyenne des groupements varie de $2,35 \mathrm{~m}^{2} / \mathrm{ha}$ dans le groupement à Prosopis africana et Pennisetum polystachion à $8,44 \mathrm{~m}^{2} / \mathrm{ha}$ dans le groupement à Daniellia oliveri et Leersia hexandra. La densité moyenne est comprise entre 95 et 275 tiges par hectare et l'indice de diversité de Shannon varie de 0,49 à 2,06 bits.

Tableau 4 : Caractéristiques structurales des groupements ligneux.

Pour les abréviations $\mathrm{A}$ à $\mathrm{H}$, voir les figures 4 et 5 . $\mathrm{G}$ surface terrière, $\mathrm{N}$ : densité, $\mathrm{H}$ : indice de Shannon

\begin{tabular}{|c|c|c|c|c|c|}
\hline Site & $\begin{array}{c}\text { Formation } \\
\text { végétale }\end{array}$ & $\begin{array}{l}\text { Groupement } \\
\text { végétal }\end{array}$ & $\mathrm{G}\left(\mathrm{m}^{2} / \mathrm{ha}\right)$ & $\mathrm{N}$ (tiges/ha) & H (bits) \\
\hline \multirow{6}{*}{ 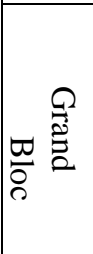 } & \multirow{3}{*}{ Savane arborée } & A & $3,46 \pm 3,14$ & $138 \pm 75$ & $1,63 \pm 0,75$ \\
\hline & & $\mathrm{B}$ & $3,31 \pm 1,87$ & $118 \pm 65$ & $1,62 \pm 0,61$ \\
\hline & & $\mathrm{C}$ & $5,21 \pm 2,11$ & $158 \pm 30$ & $1,67 \pm 0,30$ \\
\hline & \multirow{2}{*}{$\begin{array}{l}\text { Savane } \\
\text { boisée }\end{array}$} & $\mathrm{D}$ & $6,62 \pm 3,55$ & $275 \pm 145$ & $1,63 \pm 065$ \\
\hline & & $E$ & $4,37 \pm 2,41$ & $166 \pm 87$ & $1,77 \pm 0,51$ \\
\hline & Savane arbustive & $\mathrm{F}$ & $2,35 \pm 186$ & $95 \pm 91$ & $0,49 \pm 0,66$ \\
\hline \multirow{2}{*}{$\stackrel{\square}{0}$} & \multirow[b]{2}{*}{ Savane arborée } & G & $6,78 \pm 4,03$ & $225 \pm 105$ & $2,06 \pm 0,63$ \\
\hline & & $\mathrm{H}$ & $8,44 \pm 4,19$ & $140 \pm 74$ & $1,84 \pm 0,67$ \\
\hline
\end{tabular}

\section{Discussion}

\subsection{Flore et végétation du site de Ouoghi}

$\mathrm{Au}$ total, 246 espèces sont récoltées sur le site de Ouoghi sur 2807 espèces que compte la flore du Bénin, soit un taux de $8,76 \%$ (Akoègninou et al., 2006). Cette diversité floristique est assez importante pour une superficie d'environ 8 hectares car elle se regroupe en 178 genres répartis en 63 familles. La richesse spécifique de la réserve forestière de Ouoghi est inférieure à celle de 272 espèces obtenue par Sego (2008) dans la forêt communautaire de Tobé (Centre-Bénin). Toutefois, elle est supérieure à celle de 78 espèces inventoriées dans la forêt communautaire de la Basse vallée de l'Ouémé (Sud-Est Bénin) (Ali et al., 2014). Les familles les plus importantes au niveau du site sont les Leguminosae $(21,13 \%)$, les Poaceae et les Asteraceae (8,53\% chacune) et les Rubiaceae (5,69\%). Elles constituent aussi les familles les plus importantes au sein de tous les groupements végétaux. La prédominance de ces familles n'est pas une particularité du site de Ouoghi, mais une caractéristique générale de la flore du Bénin. En effet, selon Akoègninou et al., (2006), les six familles les plus riches en espèces au Bénin sont les Leguminosae (14,8\%), les Poaceae $(9,3 \%)$, les Rubiaceae et les Cyperaceae (5\% chacune), les Asteraceae $(4,6 \%)$ et les Euphorbiaceae $(4,3 \%)$. Le pourcentage des Leguminosae $(21,13 \%)$ est supérieur à ceux de $16,4 \%$ et de $15,5 \%$ obtenus respectivement par Oumorou (2003) sur les 
inselbergs du centre et du Nord Bénin et par Yédomonhan et al. (2008) sur les inselbergs du secteur méridional du Centre-Bénin. Par contre, le taux des Poaceae $(8,53 \%)$ est très proche de celui de $7,1 \%$ signalé par Yédomonhan et $a l$. (2008) et reste inférieur à celui de 11,6\% enregistré par Oumorou (2003). Parmi les principales espèces du site quatre espèces à savoir: Afzelia africana, Borassus aethiopum, Pterocarpus erinaceus et Vitellaria paradoxa figurent sur la liste des 106 espèces de plantes menacées de disparition au Bénin (Adomou et al., 2011). La forte présence-abondance des espèces telles que Azadirachta indica, Hyptis suaveolens, Imperata cylindrica, Pennisetum polystachion et Triumfetta rhomboidea est liée à l'état de dégradation poussée (formations anthropisées, surpaturâge) dans lequel se trouve chaque bloc et montre que la végétation du site est au début de sa restauration écologique et de sa dynamique vers l'état climacique (forêt dense sèche).

Huit groupements végétaux appartenant à trois formations végétales ont été décrits sur la base des espèces fréquentes et dominantes. Les formations végétales sont représentées par la savane boisée, la savane arborée et la savane arbustive. Elles présentent des groupements végétaux dont plusieurs d'entre eux sont similaires à des groupements décrits dans les travaux antérieurs (Adjakidjè, 1984 ; Trékpo, 1999 ; Yédomonhan et al., 2008). En effet, la savane boisée à Daniellia oliveri et Chromolaena odorata présente plus ou moins les mêmes caractéristiques que la savane arborée à Daniellia oliveri et Parkia biglobosa du socle cristallin décrites par Adjakidjè (1984). On note la dominance des espèces comme Daniellia oliveri, Vitellaria paradoxa et Hymenocardia acida. La savane boisée à Isoberlinia doka et Pennisetum polystachion a une composition floristique qui s'apparente au groupement à Isoberlinia doka et Isoberlinia tomentosa de Trékpo (1999) dans la forêt classée Ouenou-Benou au Nord-Est avec des espèces dominantes comme Isoberlinia doka, Pterocarpus erinaceus, Burkea africana, Daniellia oliveri et Vitellaria paradoxa. Cette formation est aussi semblable à la savane arborée à Isoberlinia doka et Andropogon tectorum décrit par Yédomonhan et al. (2008) sur les inselbergs du secteur méridional du centre Bénin.

Les spectres biologiques sont dominés par les phanérophytes dans tous les groupements végétaux (45,97 à 67,35\%). Ces résultats sont conformes à ceux de Ali et al. (2014) et de Yédomonhan et al. (2008) obtenus dans les groupements de formations ligneuses. Le fort pourcentage de ces phanérophytes dans les formations végétales est dû aux jeunes individus des espèces ligneuses et aussi à des individus adultes de certaines espèces à valeur socio-économique importante comme Vitellaria paradoxa. $\mathrm{Au}$ plan phytogéographique, les espèces à large distribution prédominent au niveau de tous les groupements sauf dans le groupement à Daniellia oliveri et Leersia hexandra du petit bloc où elles laissent place aux espèces 
soudano-guinéennes. Ceci s'explique, soit par le microclimat d'humidité du sol engendré, soit par son positionnement au bas de pente, soit épargné lors des défrichages et des coupes de bois au cours de l'exploitation du site. La variation du spectre biogéographique d'un groupement à un autre a été déjà observée par d'autres auteurs, pour qui les spectres biogéographiques dépendent des types de formation végétale (Guinko, 1984 ; Yédomonhan, 2002). Toutefois, La contribution relativement élevée (34,64 à 39,83\%) des espèces à large distribution au sein de la plupart des groupements met en exergue que le site avait subi une très forte pression anthropique d'une part et sa végétation provient d'une longue friche d'autre part.

\subsection{Caractéristiques structurales du peuplement ligneux du site}

Selon les travaux de Malaisse (1982), le recouvrement basal est de 1 à $5 \mathrm{~m}^{2} /$ ha en savane arborée. Seule la savane arborée du petit bloc présente des groupements dont les peuplements ligneux ont des surfaces terrières qui dépassent $\left(6,78\right.$ et $8,44 \mathrm{~m}^{2} /$ ha) nettement cette norme. Cependant, ceci ne traduit pas une bonne structuration naturelle, mais il est simplement lié au fait que c'est dans ces groupements que figurent nombreux individus de Daniellia oliveri et de Terminalia glaucescens.

La densité des arbres et arbustes des groupements varie de 95 à 275 tiges/ha. Elle est inférieure à celle de 206 à 1333 tiges/ha obtenue sur les inselbergs du secteur méridional du Centre-Bénin (Yédomonhan et al., 2008). Par contre, le groupement à Daniellia oliveri et Andropogon tectorum et le groupement à Daniellia oliveri et Chromolaena odorata ont respectivement une densité de 225 tiges/ha et 275 tiges/ha qui est bien située dans fourchette de 167 à 1947 tiges/ha pour les forêts néotropicales (Gentry, 1982).

Suivant les échelles de Frontier et Pichod-viale (1995), les valeurs de l'indice de diversité de Shannon montrent que les huit groupements végétaux sont faiblement diversifiés. Ceci se justifie par la prédominance d'une espèce sur les autres. De plus, la réserve forestière de Ouoghi est une ancienne implantation de l'Institut de Recherche pour les Huiles et Oléagineux (IRHO) dont la végétation a été fortement dégradé en faveur de la production du ricin (Ricinus communis).

\section{Conclusion}

Cette étude est un état de lieu du site de Ouoghi situé dans la zone soudano-guinéenne en vue de la création d'une réserve forestière. Sur le plan floristique, le site de Ouoghi renferme 246 espèces dont 169 au niveau du grand bloc et 107 pour le petit bloc. Ces espèces sont réparties dans 178 genres et 63 familles dont les plus importantes sont les Leguminosae $(21,13 \%)$, les Asteraceae et Poaceae (8,53\% chacune) et les Rubiaceae 
(5,69\%). Cette diversité floristique est dominée par les phanérophytes d'une part puis les espèces à large distribution d'autre part.

Sur le plan physionomique, huit groupements végétaux répartis dans trois formations végétales ont été identifiés. Il s'agit de la savane arbustive, de la savane arborée et de la savane boisée. La composition floristique et la structure du site témoignent de la nécessité de sa protection intégrale pour sa dynamique vers une végétation climacique. Par conséquent, tout aménagement visant une production de bois lui sera préjudiciable. L'objectif des aménagements possibles du site doit viser la sauvegarde de la biodiversité par la protection et la reconstitution de la végétation naturelle. Cela ne peut toutefois être atteint que si les populations riveraines sont associées pour une gestion participative.

\section{Remerciements}

Cette étude a bénéficié d'un appui financier de l'Ambassade de France près le Bénin et du Centre de Recherches Agricoles du Centre-Bénin (CRA-Centre) à qui les auteurs expriment leur profonde gratitude.

\section{References:}

1. Achard F., Eva H. D., Stibig H. J., Mayaux P., Gallego J., Richards T. \& Malingreau J.-P. 2002. Determination of deforestation rates of the world's humid tropical forests. Science 297 : 999-1002

2. Adjakidjè V. 1984. Contribution à l'étude botanique des savanes guinéennes de la République populaire du Bénin. Thèse de doctorat de 3ème cycle, Université de Bordeaux III, 285 p.

3. Adomou A. C., Akoègninou A., Sinsin B., de Foucault B. \& van der Maesen L. J. G. 2007. Biogeographical analysis of the vegetation in Bénin. Acta Bot. Gallica, 154 (2): 221-233.

4. Adomou A. C., Agbani O. P. \& Sinsin B. 2011. Plantes. In Protection de la Nature en Afrique de l'Ouest: Une Liste Rouge pour le Bénin. Nature Conservation in West Africa: Red List for Benin, Neuenschwander P, Sinsin B, Goergen G (eds). International Institute of Tropical Agriculture: Ibadan, Nigeria; 21-46.

5. Agbo V. \& Sokpon N. 1997. Forêts sacrées et patrimoine vital au Bénin. Rapport technique

6. provisoire, $200 \mathrm{p}$.

7. Akoègninou A. 2004. Recherches botaniques et écologiques sur les forêts actuelles du Bénin. Thèse d'état, Université de CocodyAbidjan (Côte d'Ivoire), $326 \mathrm{p}$.

8. Akoègninou A., van der Burg W.J. \& van der Maesen L. J. G. 2006. Flore analytique du Bénin. Backhuys Publishers, Wageningen, 1034 p. 
9. Ali R. K. F. M., Odjoubere J., Tente A. B. H. \& Sinsin A. B. 2014. Caractérisation floristique et analyse des formes de pression sur les forêts sacrées ou communautaires de la Basse Vallée de l'Ouémé au Sud-Est du Bénin. Afrique Science 10 (2) : 243- 257.

10. Amadou Siako. A. S. 2003. Utilisation agro-pastorale des terroirs riverains au Parc National du W : Axe Kérou-Rivière Mékrou au Nord du Bénin dans la commune de Banikoara. Mémoire de maîtrise en Géographie Physique, FLASH/UAC, 102 p.

11. Amahowé O. I. 2003. Contribution à l'aménagement durable des forêts villageoises de Glazoué. Mémoire du Diplôme d'Ingénieur des Travaux, EPAC/UAC, 162 p.

12. Aprofond-Bénin. 2000. Conservation et aménagement de la biodiversité de la forêt sacrée des singes noirs et blancs de Kilélé, Commune de Bassila, Département de la Donga. UICN/CBDDAPROFOND-BÉNIN, Kilélé, Bassila, Bénin. 16 p.

13. Arbonnier M. 2009. Arbres, arbustes et lianes des zones sèches d'Afrique de l'Ouest. Edition Quae, MNHN, 573 p.

14. Braun-Blanquet J. 1932. Plant sociology: The study of plant communities. McGray Hill, NewYork and London, 439 p.

15. Daget P. 1980. Le nombre de diversités de Hill, un concept unificateur dans la théorie de la

16. diversité écologique. Acta Oecological/Oecol. Gener. 1 (1): 51-70

17. DGRN 2009. Répartition des forêts classées par Département. Rapport, 2 p.

18. Dubroeucq D. 1977. Carte pédologique de reconnaissance de la République Populaire du Bénin à 1/200 000 : feuille Parakou. ORSTOM, notice explicative 66 (5), $37 \mathrm{p}$.

19. FAO 2005. Evaluation des ressources forestières mondiales 2005. FAO Etude forêt. Rome,

20. Italie.

21. FAO 2010. Evaluation des ressources forestières mondiales 2010. Rapport national, Bénin : FRA 2010. Rome, 54 p.

22. Faure P. 1977. Carte pédologique de reconnaissance de la République Populaire du Bénin à

23. 1/200 000 : feuille Djougou. ORSTOM, notice explicative 66 (4), 49 p.

24. Frontier S. \& Pichod-Viale D. 1991. Ecosystèmes: structure, fonctionnement, évolution. Masson, Paris - France. Collection d'écologie $21: 392 \mathrm{p}$.

25. Frontier S. \& Pichod-Viale D. 1995. Théorie des écosystèmes : structure, fonctionnement, évolution, 2e édition. Paris, Masson. Collection d'écologie 21: 287-311. 
26. Ganglo C. J. 1999. Phytosociologie de la végétation naturelle de sous-bois, écologie et productivité des plantations de teck (Tectona grandis L.f.) du sud et du centre-Bénin. Thèse de doctorat, Université Libre de Bruxelles, Section Interfacultaire d'Agronomie, Laboratoire de Botanique, Systématique et de Phytosociologie, $391 \mathrm{p}$.

27. Gentry A. H. 1982. Patterns of neotropical plant species diversity. In : M.K. Hecht, B. Wallace \& G.T. Prance ((eds.) Evolutionary biology 15 : 123-135. New-York, Plenum Press.

28. Guinko S. 1984. Végétation de la Haute Volta. Thèse de Doctorat d'Etat ès-Sciences Naturelles, Bordeau III, 394 p.

29. Hendeson P. A. \& Seaby R. M. 2002. Community Analysis Package 2.15 (CAP). Ltd, IRC

30. House, Pennington, Lymington, SO41 8 GN, UK.

31. Heymans J. C. \& Pétit J. M. 1985. Etude et aménagement de la Forêt Classée des Monts Kouffé (Rép.Pop. du Bénin). Note préliminaire. Tropicultura 3 (3) : 88-92

32. INSAE 2015. Quatrième recensement général de la population et de l'habitat: que retenir des effectifs de population en 2013, MDAEP, Cotonou, Bénin. $35 \mathrm{p}$

33. Legendre L. et Legendre P. 1984. Ecologie numérique 2 - La structure des données écologiques. Masson collection d'écologie 13, $335 \mathrm{p}$.

34. Malaisse F. 1982. Comparaison de la structure ligneuse dans une série régressive de la région zambézienne : forêt dense sèche - forêt claire - savane boisée (Luiswishi, Shaba, Zaire). Geo-Eco-Trop, 6 (4) : 309 - 350 .

35. Myers N., Mittermeier R. A., Mittermeier C. G., da Fonseca G. A. B. \& Kent J. 2000. Biodiversity hotsposts for conservation priorities. Nature 403: 853-858.

36. Oumorou M. 2003. Etudes écologique, floristique, phytogéographique et phytosociologique des inselbergs du Bénin. Thèse de doctorat unique en Sciences, Université Libre de Bruxelles, $210 \mathrm{p}$.

37. Raunkiaer C. 1934. The life forms of plants and statistical plant geography. Clarendron Press, London, $632 \mathrm{p}$.

38. Sokpon N. 1995. Recherche écologique sur la forêt dense semidécidue de Pobè au Sud Bénin : groupements végétaux, structure, régénération naturelle et chute de litière. Thèse de doctorat, Université Libre de Bruxelles (Belgique), 365 p.

39. Trékpo P. 1999. Contribution à l'étude des possibilités d'aménagement durable de la forêt 
40. classée de Ouenou-Benou au Nord-Est du Bénin : structure et dynamique des principaux groupements végétaux. Mémoire d'ingénieur des travaux, CPU/UNB, $144 \mathrm{p}$.

41. Ségo A. 2008. Phytodiversité et conservation de la forêt communautaire de Tobé dans la

42. commune de Bantè (Republique du Bénin). Mémoire d'ingénieur des travaux, EPAC /UAC, $82 \mathrm{p}$.

43. White F. 1983. The vegetation map of Africa. A descriptive memoir, UNESCO. Natural resources Reserch $20: 1-356$.

44. Yédomonhan H. 2002. Etude des formations saxicoles du secteur méridional du Centre Bénin. Mémoire de Maîtrise de Géographie, FLASH/UAC, $95 \mathrm{p}$.

45. Yédomonhan H., Hounadagba C. J., Akoègninou A. \& van der Maesen L. J. G. 2008. Structure et diversité floristique de la végétation des inselbergs du secteur méridional du Centre-Bénin. Syst. Geogr. Pl. 78 : 111-125. 